Cahiers de recherches médiévales

\title{
Pierre de Provence et la réception des romans de chevalerie médiévaux dans la France du XVII ${ }^{e}$ siècle
}

Helwi Blom

\section{(2) OpenEdition \\ 12 Journals}

Édition électronique

URL : https://journals.openedition.org/crm/2481

DOI : 10.4000/crm.2481

ISSN : 1955-2424

Éditeur

Honoré Champion

Édition imprimée

Date de publication : 12 décembre 1996

Pagination : 51-60

ISSN : 1272-9752

\section{Référence électronique}

Helwi Blom, «Pierre de Provence et la réception des romans de chevalerie médiévaux dans la France

du XVII siècle », Cahiers de recherches médiévales [En ligne], 2 | 1996, mis en ligne le 04 février 2008, consulté le 15 décembre 2022. URL : http://journals.openedition.org/crm/2481 ; DOI : https://doi.org/ $10.4000 / \mathrm{crm} .2481$ 


\section{Pierre de Provence et la réception des romans de chevalerie médiévaux dans la France du XVII ${ }^{e}$ siècle}

En 1665, dans son Idee de la ville de Montpellier, Pierre Gariel, doyen des chanoines de l'église cathédrale de cette ville, parlant du comte Petrus de Mergueil et son épouse Aldemunde dont les signatures figuraient en bas d'un acte de 1079, observa: «Cette treschere, \& tres-aymée Aldemunde, ou Almunde, est la belle Italienne Magalone, \& son doux Amy Pierre est le Comte. Et ainsi ce sont les deux renommez Amans qui ont fourny le sujet, \& la matiere de ce vieux Roman qui court encore parmy le peuple. $\rangle^{1}$ Ce témoignage est très intéressant, non seulement parce qu'il comprend un essai d'identifier les protagonistes de l'histoire de Pierre de Provence à des personnages historiques, mais il est également fascinant du point de vue de l'histoire de l'édition et de la réception d'œuvres littéraires du Moyen Age. C'est cette question de la fortune de la littérature médiévale aux temps modernes qui retiendra notre attention dans les pages qui suivent; à partir de l'exemple du roman de Pierre de Provence ${ }^{2}$ nous étudierons quelques aspects de la réception de la littérature chevaleresque du Moyen Age à une époque considérée généralement comme son antipode même, le Grand Siècle. Nous envisagerons ce sujet sous deux angles, à savoir celui de la réception matérielle de Pierre de Provence, c'est-à-dire les réeditions qui ont paru au XVII s., et celui de sa réception productive, à savoir les allusions à Pierre et Maguelonne dans la littérature du Grand Siècle.

La réception matérielle de Pierre de Provence au $\mathrm{XVI}^{\mathrm{e}}$ s.; les éditions de la Bibliothèque bleue

Plus de trente ans après la parution de la première édition du livre de R. Mandrou sur la culture populaire aux XVIf et XVIII siècles ${ }^{3}$, peu de gens ignorent que, du XVII au XIXe siècle, bon nombre de textes médiévaux ont sans cesse été réédités à Troyes et à Rouen par les soins des éditeurs de la Bibliothèque bleue. Comme beaucoup d'autres romans chevaleresques du Moyen $\mathrm{Age}^{4}$, Pierre de Provence aussi entra dans cette fameuse "collection", qui, selon les spécialistes, se caractérise surtout par ses aspects matériels: caractères et bois usés, typographie peu soignée, mauvais papier, couverture de papier coloré etc. Ces caractéristiques indiquent que la Bibliothèque bleue est en fait une formule éditoriale utilisée pour rëimprimer en grand et à bon marché des ouvrages à succès. Bien que plusieurs chercheurs aient attiré l'attention sur le fait que le troyen Nicolas Oudot I, considéré

\footnotetext{
${ }^{1}$ P. Gariel, Idée de la ville de Montpellier, recherchée et presentée aux honestes gens, Montpellier, 1665, p. 129.

${ }^{2}$ Un résumé de ce roman bien connu se trouve dans le Dictionnaire des lettres frangaises. Le Moyen Age. Edition entièrement revue et mise à jour sous la direction de G. Hasenohr et M. Zink, Paris, 1992, pp. 1191-1192.

3 R. Mandrou, De la culture populaire aux Xuf et XVIff siècles. La Bibliothèque bleue de Troyes, Paris, Stock, 1964.

${ }^{4} J e a n$ de Paris, Mélusine, Les Quatre fils Aymon, Richard sans Pewr, Valentin et Orson, Galien restauré, Ogier le Danois pour ne citer que quelques exemples.
} 
généralement comme le patriarche de la Bibliothèque bleue, a eu des précurseurs, notamment à Lyon et à Paris, l'idée que les premiers livres bleus ont paru à Troyes au début du XVII's. n'a pas vraiment été mise en question. ${ }^{5}$

Une large partie des études menées sur les livrets bleus signale la mauvaise qualité non seulement du papier, des caractères et des illustrations utilisés, mais aussi de la composition des textes eux-mêmes. Pourtant le nombre d'analyses systématiques de l'évolution des textes chevaleresques bleus à travers les âges se compte sur les doigts d'une main, et le problème compliqué des relations entre les éditions "bleues" et leurs modèles n'a guère reçu d'attention de la part des chercheurs ${ }^{6}$. Ce problème touche cependant le cour du concept de la Bibliothèque bleue, car il met en avant des questions concernant ses origines et sa définition. II ne peut évidemment pas être question d'aborder un si vaste sujet dans cet article; nous décrirons ci-dessous plusieurs éditions différentes de Pierre de Provence, en insistant sur un certain nombre de correspondances et divergences remarquables. Ensuite nous confronterons nos résultats à la définition courante de la Bibliothèque bleue.

Nous avons repéré douze éditions de Pierre de Provence datant du Xvir siècle ${ }^{7}$. Bien que ce nombre, qui ne représente sans doute qu' une partie des éditions réalisées à l'époque $\mathbf{8}^{\mathbf{8}}$, ne lui vaille qu'un rang modeste sur la liste des romans de chevalerie les plus souvent

${ }^{5}$ Cf. e.a. H.-J. Martin, “ Culture écrite et culture orale. Culture savante et culture populaire dans la France d'Ancien Régime », Joumal des Savants, 1975, pp. 225-282 ; J.P. Oddos, "Simples notes sur les origines de la Bibliothèque bleue ", $L a$ "Bibliothèque bleue " nel seicento o della letteratura per il popolo, Bari/Paris, 1981, pp. 159-168 ; M.-D. Loclerc et A. Robert, Des éditions au succès populaire ; les livrets de la Bibliothèque bleue, Xvif-XxX' siècles. Présentation-anthologie-catalogue, Troyes, Centre dép. de documentation pédagogique, 1986 ; R. Chartier, «Trajectoires et tensions culturelles de l'Ancien Régime », Histoire de la France : Les formes de la culture, dir. A. Burguière et J. Revel, Paris, 1993, pp.307-392. ; M.D. Leclerc. "L'Histoire des Quatre fils Aymon Essai d'analyse iconographique ", La vie en Champagne, 4, 1995, pp. 3-36 ; A. Robert "Aux origines de la Bibliothèque bleue. Quelques réflexions sur la production de Nicolas Oudot », Le Bibliophile Rémois, 39, 1995, pp. 3-5.

${ }^{6} \mathrm{~A}$ notre connaissance seuls A. Chassagne-Jabiol, Evolution d'un roman médiéval à travers la littérature de colportage : "La Belle Hélène de Constantinople ", XVf-xxx siécles, thèse inédite, Ecole des Chartes, 1974 ; H.J. Martin, art cit, et J.-P. Oddos, art cit, se sont occupés, de façon plus, ou moins approfondie, de la question des modèles utilisés par ceux qui, au XVI" $\mathrm{s}$., ont donné des éditions 'bleues'de romans de chevalerie.

${ }^{7}$ 1. Paris, Nicolas et Pierre Bonfons, [v. 1600], 47 pp. in- $4^{\circ}$. 2. Rouen, Louis Costé, s.d., in- $4^{\circ}$. 3. Lyon, Jean Didier, 1625, 64 pp. in $-8^{\circ}$. (Paris, Ars., $8^{\circ}$ BL 17295) 4. Lyon, Claude Chasteland, 1630, in $-8^{\circ} .5$. Rouen, David Ferrand, s.d. [1ère moitié du siècle], 48 pp. (Evanston, Northwestem University, 843.2. J 428 F) 6. Troyes, Nicolas Oudot, s.d., 64 pp. in- $8^{\circ}$. (Paris, B.N., Rés. Y2 3142) 7. Lyon, Claude Chancey, 1665, 64 pp. in- $8^{\circ}$. (Paris, B.N., Rés. p. Y2 3048 (3)) 8. Troyes, Jacques Oudot, 1687, in-8 (Montpellier, B.M.) 9. Troyes \& se vend à Paris chez Antoine de Rafflé, s.d. [2e moitié du siècle], 64 pp. in- $8^{\circ}$. (Evreux. B.M., (m) 1201, pièce 1 ; Oxford, Bodleian Library, Douce R 317 (5) ; New York, Columbia University, Spec.colls. B 84 P 615 I C) 10. Troyes \& se vendent à Paris, chez Jean Musier, s.d. [entre 1696 et 1703 ], 64 pp. in- $8^{\circ}$. (Austin, University of Texas, 398.5 F 887 Rare books coll.) 11. Troyes, Jacques Oudot, 1700, 64 pp. in- $8^{\circ}$. (Paris, Bibl. du Musée des ATP, 1 R.1274) 12 Rouen, JeanBaptiste Besongne, 1700,48 pp. in- $8^{\circ}$. (Paris, Ars., $8^{\circ}$ B 28838)

${ }^{8}$ Le fait que de trois de nos éditions aucun exemplaire n'a été conservé et de huit autres pas plus qu'un seul exemplaire, est une preuve indubitable des maigres chances de survie des éditions de Pierre de Provence réalisées il y a plus de trois cents ans. 
réédités", il témoigne bien de la popularité dont jouissaient Pierre et Maguelonne. N'oublions d'ailleurs pas que, si la mauvaise qualité des éditions «bleues" les destinait déjà à une vie de courte durée, le format et le volume des éditions de petits romans comme Pierre de Provence (les éditions les plus volumineuses comptent 64 pages in-89) augmentaient encore leur risque de se perdre dans la nuit des temps. Nos éditions, comme plus de $95 \%$ des 270 éditions de romans chevaleresques médiévaux publiées au Grand Siècle figurant sur notre fichier, ont été réalisées dans les trois villes de Troyes, Rouen et Lyon. Elles ont probablement toutes porté les caractéristiques d'une production à bon marché. Il en est en tout cas ainsi pour les exemplaires que nous avons eus sous les yeux. Celles publiées à Troyes et à Rouen peuvent sans aucun doute être qualifiées comme éditions "bleues", imprimées par les soins des éditeurs célèbres de la Bibliothèque bleue et leurs concurrents. Mais en ce qui conceme les éditions lyonnaises, l'emploi de l'étiquette «bleue» pose des problèmes. En effet, à l'exception de L. Andries qui a inséré certaines publications d'éditeurs lyonnais exerçant entre 1600 et 1630 dans sa liste de livres de la Bibliothèque bleue ${ }^{10}$, d'ailleurs sans justifier cela dans son texte, aucun spécialiste de la «collection " ne l'a mise en rapport avec des éditeurs lyonnais du XVur siècle. Or, nous sommes d'avis que les quelques éditions chevaleresques lyonnaises figurant sur la liste établie par L. Andries, parmi lesquelles se trouve aussi l'édition Chastelard de Pierre de Provence, appartiennent en effet à la Bibliothèque bleue, et pas seulement elles, mais bon nombre d'autres livres lyonnais, parmi lesquels les éditions de notre histoire données par Didier et par Chancey. C'est que les aspects matériels d'un certain nombre d'éditions lyonnaises du XVII ${ }^{e} \mathrm{~s}$. ressemblent beaucoup à ceux que présentent les éditions «bleues " des mêmes titres publiées à Troyes et à Rouen. Et, qui plus est, plusieurs inventaires de libraires lyonnais de la deuxième moitié du siècle font mention de "papier bleud ". " Nous voudrions donc lancer l'hypothèse que, du début jusqu'à la fin du XVI' s., Lyon a joué un rôle non négligeable sur le plan de l'édition de livres de la Bibliothèque bleue, ou du moins de romans de chevalerie «bleus ». Il n'est pas possible d'élaborer ici notre hypothèse, mais soulignons dès maintenant le fait qu'elle soulève des questions nouvelles sur les origines et la définition de la «collection bleue ». ${ }^{12}$

Dans l'histoire de Pierre de Provence les protagonistes vivent maintes aventures périlleuses. Une lecture comparative d'un certain nombre d'éditions différentes du roman nous apprend que le texte lui-même a souffert bon nombre de tribulations ${ }^{13}$. Les premières

\footnotetext{
${ }^{9}$ Les romans les plus populaires au XVII s. étaient Les Quatre fils Aymon, Valentin et Orson, et Huon de Bordeaux. Nous avons repéré une vingtaine d'éditions de chacun de ces trois textes.

${ }^{10} \mathrm{Cf}$. L. Andries, La Bibliotheqque bleue au dix-huitième siècle : une tradition éditoriale, Oxford, The Voltaire Foundation, 1989.

"Citons ici seulement l'exemple de l'imprimeur et libraire Pierre Bailly qui au moment de sa mort avait 33 rames de papier bleu en stock. Cf. Archives départementales du Rhône, B.P. 1925, inventaire du 1803-1655 et jours suivants.

${ }^{12}$ Cette question sera traitée dans notre thèse, consacrée à la présence des romans de chevalerie médiévaux dans la France du XVư siècle. Nous espérons soutenir cette thèse en 1998 à l'Université d'Utrecht.

${ }^{13}$ Pour notre étude comparative nous avons dû nous baser sur des éditions dont des exemplaires étaient disponibles dans les bibliothèques parisiennes, à savoir, pour le $\mathrm{Xv}^{f}$ s., les éditions de Guillaume Le Roy, Pierre Bouteiller, Jean de La Fontaine et Jean Trepperel, pour le XVI ${ }^{e}$ s., celle de la vearve Trepperel, et pour le XVII s., celles de Didier, Chancey, Besongne, N. Oudot et J. Oudot. Rappelons que, à l'exception de l'édition de B. Buyer ( $\left.x v^{e} s.\right)$, toutes les éditions connues sont basées sur la version courte de notre histoire, donnée par le manuscrit de Cobourg.
} 
éditions, donnant la version du manuscrit de Cobourg, ont paru à Lyon et datent des années 1480. Elles offrent au lecteur un texte soigné, proche du manuscrit, imprimé en caractères gothiques, souvent avec des encres de couleurs différentes, sur un papier de bonne qualité, plié in-folio ou in-quarto, et illustré de belles lettrines et bois (coloriés), conçus spécialement pour omer notre roman. A partir du $X^{e} v^{e} s$., la qualité des éditions, toutes de format inquarto, imprimées en noir, et illustrées de bois qui sont des imitations peu fines de bois du $\mathrm{XV}^{E}$ s., va en s'amoindrissant. Malheureusement, très peu d'éditions du $X v^{\complement} \mathbf{s}$. ont été conservées $^{14}$, ce qui, comme on le verra, rend impossible de bien suivre l'évolution du texte, et d'attribuer avec certitude à tel ou tel éditeur l'origine des adaptations ou mutilations que présentent les éditions du XVü siècle.

Les éditions de notre histoire publiées au Xvur s. paraissent être issues de deux traditions éditoriales différentes. Celles de Lyon copient assez fidèlement les publications réalisées un ou deux siècles auparavant par des imprimeurs lyonnais. Mais le format de leurs impressions - dont dépend la quantité de papier nécessaire - est plus réduit (in-8 ${ }^{\circ}$ ), la typographie est mauvaise, ainsi que la qualité du papier, surtout celle de l'édition Chancey, et la pagination est souvent fautive. Quant aux illustrations, si l'édition Didier contient quelques petits bois représentant des scènes que l'on ne retrouve pas dans le texte, celle publiée par Chancey ne donne qu'une petite gravure sur la page de titre. Malgré leur fidélité relative au texte des éditions lyonnaises du $\mathrm{XV}^{e}$ (et $\mathrm{XVI}^{\mathrm{e}}$ ?) siècles, les éditions de Didier et Chancey présentent des exemples frappants des transformations qu'a subies le roman de Pierre de Provence au cours des siècles. Dans le manuscrit de Cobourg et dans les éditions lyonnaises du XV ${ }^{\varepsilon}$ s., Pierre remercie Maguelonne de l'invitation à venir souvent à la cour en utilisant le titre «vostre seigneurie»; dans le texte de Didier, Pierre adresse ses remerciements à « Nostre Seigneur ». Dans cette même édition, la fin du chapitre qui raconte la première rencontre des protagonistes est amputée de quelques lignes, soit à cause d'un désir d'abréger le texte, soit - et cela nous parait plus probable - à cause du fait que celui qui a composé le texte par accident a sauté quelques lignes; selon les premières versions lyonnaises la Reine, Maguelonne et leurs dames et «damoiselles» rentrent dans leurs chambres, tandis que le Roi et les chevaliers restent encore dans la salle, mais dans l'édition Didier quelques phrases ont dispanu, de sorte que les dames retournent à leurs logis « avec les seigneurs ». Ensuite la scène de la rencontre de Pierre et Maguelonne dans la chambre de cette demière, rencontre lors de laquelle Maguelonne « changea de couleur» et Pierre «pas moins ", a perdu un peu de sa force dans la version de 1625 , où nous lisons que Pierre "ne changea point de couleur", pendant que Maguelonne le regardait "pieusement" au lieu de «piteusement ». L'édition de Chancey (1665) présente à peu près les mêmes particularités et en ajoute d'autres encore. Ainsi ce ne sont plus les (housses des) «chevaux» de Pierre qui sont "couverts de clefs" en l'honneur de Saint Pierre, mais ce sont ses "chariots», et la durée de la fête après le mariage des deux amants, vingt-deux jours, est réduite à deux jours,

\footnotetext{
${ }^{14}$ Ont été perdues les éditions de B. Chaussard et sa veuve (Lyon, début XVI s.), celle de J. de Channey (Avignon, 1524), celles de J. Le Forestier et R. Gaultier (Rouen, 1516), a finalement les éditions parisiennes de M. le Noir (1504), de la veuve J. Bonfons (vers 1570), deux éditions de son fils Nicolas, et une édition réalisée par Nicolas avec son fils Pierre à la fin du XVI', ou au début du XVI ${ }^{\mathrm{e}}$ siècle. L'exemplaire de l'é-dition de J. Bonfons qui est conservé au Musée Condé n'a pas pu être consulté, le musée étant fermé pendant notre séjour à Paris.
} 
probablement grâce à un compositeur négligent. En outre la ponctuation de cette édition est détériorée par rapport à celle de Didier (1625).

Les éditions de la deuxième tradition éditoriale, celles réalisées à Troyes et à Rouen, donnent des versions qui diffèrent sensiblement des publications lyonnaises, bien qu'elles aient les mêmes caractéristiques matérielles: format in $-8^{\circ}$, papier de mauvaise qualité, et une pagination qui, si elle ne fait pas défaut, laisse beaucoup à désirer, ainsi que la ponctuation ; si les textes sont déjà illustrés, les bois n'ont peu ou pas de rapport avec l'histoire. Par exemple, le chevalier Ferrier de la Couronne, originaire de Rome (ou du pays de Romanie) selon les éditions lyonnaises, vient de Normandie. Et, contrairement aux éditions de Didier et de Chancey et leurs prédécesseurs, qui avaient pris soin de noter pour chaque prière ou pensée religieuse que Pierre et/ou Maguelonne s'adressaient non seulement à Dieu mais aussi à la Vierge et à Saint Pierre, les éditions troyennes et rouennaise du XVI ${ }^{e}$ s. ont omis presque chaque fois les interpellations des deux derniers. La division en chapitres ne correspond non plus à celle de la plupart des éditions de la tradition lyonnaise. Nous pensons que les éditions de Nicolas et de Jacques Oudot, ainsi que celle de Jacques Besongne, ne descendent pas des éditions lyonnaises, mais d'une édition parisienne ${ }^{15}$ datant du XVI ${ }^{e}$ siècle. C'est que, en dépit du fait que les éditions parisiennes que nous avons pu étudier sont encore conformes aux éditions de Lyon, pour ce qui regarde les lectures de Romanie/Normandie et les prières, elles présentent, quant à la division du texte en chapitres, les mêmes caractéristiques que les éditions troyennes du XVI' s., avec lesquelles elles ont encore en commun plusieurs autres particularités, comme nous le verrons.

La plus vieille édition troyenne, dont nous ne saurions dire si elle est sortie des presses de Nicolas Oudot I (ex. vers 1600-vers 1635) ou de celles de son fils Nicolas II (ex. vers 1640 vers 1688), donne souvent les leçons du manuscrit de Cobourg à des endroits où les éditions lyonnaises ne le font pas ; mais à beaucoup d'autres endroits, l'édition Nicolas Oudot, pleine de fautes de ponctuation, omet des phrases ou les mutile de telle façon que le texte devient incompréhensible. Par exemple, comment interpréter les mots suivants, adressés par la nourrice à Maguelonne : «... les choses qui so[n]t faictes \& ordonnées par volonté ne sont pas à l'honneur de ceux qui les fo[n]t mespriser de ceux qui ente[n]dent bien que vous l'aimez, car il est bien digne. " ? C'est seulement à l'aide d'éditions plus anciennes que nous comprenons ce que la nourrice a voulu dire : « les choses qui sont faites desordonnément par volonté hastive ne sont pas à l'honneur de ceux qui les font, ni prisées par ceux qui les entendent. Je loue bien que vous l'aimiez, car il en est bien digne, mais.... » Oudot a supprimé le monologue désespéré de Pierre découvrant que l'on l'a laissé seul dans l'île de Sangona, passage qui se trouve dans toutes les éditions lyonnaises que nous avons consultées. A première vue, il est tentant de tenir Nicolas Oudot pour responsable de cette amputation de notre roman, qui aurait èté la conséquence du besoin des éditeurs de la Bibliothèque bleue de travailler à moindres frais et d'abréger donc leurs modèles. Mais nous avons constaté que le monologue de Pierre est déjà absent de l'édition qui a été réalisée en 1493, à peine une décennie après la parution de la première édition du manuscrit de Cobourg, par l'imprimeur parisien Jean Trepperel, et également de celle publiée quelques années après par sa veuve. En effet il semble que l'édition Trepperel, qui est la plus ancienne

\footnotetext{
${ }^{15}$ Cela n'empêche pas que les éditions parisiennes aient eu pour modèle une édition lyonnaise. Nous y reviendrons.
} 
édition parisienne connue, et celle de sa veuve, représentent des étapes décisives dans la vie éditoriale de Pierre et Maguelonne, car à côté de cette omission du monologue, plusieurs autres éléments remarquables de l'édition Nicolas Oudot, comme sa division du texte en chapitres, figurent aussi dans l'une de ces deux éditions ou dans les deux. Reste à savoir si Trepperel a fondé son édition sur le manuscrit de Cobourg, ou s'il a utilisé comme modèle une des premières éditions. Nous avons l'impression qu'il s'est bien servi d'une édition existante, qui doit avoir été proche de la superbe édition publiée par le lyonnais Guillaume Le Roy vers 1485 . C'est que cette demière offre une version de notre roman qui donne à plusieurs endroits des leçons et une division des chapitres correspondant à celles des éditions de Trepperel, sa veuve, et de celles publiées au Grand Siècle à Troyes et à Rouen. Ce sont justement ces traits-là qui différencient cette version et une édition lyonnaise sans date $\left(x v^{e}\right.$ s.), attribuée à Pierre Bouteiller, et une autre, de 1491, qui a probablement été réalisée par le lyonnais Jean de La Fontaine. ${ }^{16}$ Nous pensons que l'édition de Bouteiller, qui sur plusieurs des points où elle ne suit pas l'édition Le Roy donne la même leçon que celle de 1491 et les éditions lyonnaises du $\mathrm{XVu}^{e}$ s., est postérieure à l'édition Le Roy, dont elle imite de façon un peu primitive les illustrations, et qu'elle est à la base de ce que nous appelons la " tradition lyonnaise ». Pourtant il y a des endroits où l'édition Le Roy donne la «leçon lyonnaise » et non pas celle donnée par Trepperel et ses successeurs. Nous ne savons pas si ces divergences sont dues à Trepperel ou à une édition intermédiaire. La naissance des deux traditions de notre histoire semble en tout cas bien dater des années 1485-1495. Les versions présentées par les deux traditions n'ont d'ailleurs pas cessé d'évoluer, les éditions parisiennes en omettant la Vierge et Saint Pierre des prières ${ }^{17}$ et en transformant Rome en Normandie, les éditions lyonnaises en omettant une partie du chapitre du dîner. Et dans les deux traditions il est évidemment question de fausses lectures, omissions de lignes etc. qui se multiplient. Pourtant elles gardent bien les caractéristiques, l'une de l'édition Trepperel (basée sur une édition proche de celle de Le Roy), l'autre d'une édition lyonnaise proche de celle de Bouteiller.

Un des descendants de Nicolas Oudot, Jacques Oudot, a publié en 1687 et en 1700 des éditions nouvelles de notre histoire. Celle de 1700 , comme celle de 1687 probablement, donne un texte qui ressemble beaucoup à la version donnée par son (grand-)père. Pourtant un certain nombre de «fautes " de cette dernière n'y figure pas, car tout en gardant le même nombre de pages et le même format, quoique le papier utilisé soit plus petit que celui de Nicolas Oudot, cet éditeur offre à ses lecteurs un texte radicalement reformulé et modernisé;

\footnotetext{
${ }^{16}$ Ainsi les sections sur le dîner de Pierre au palais du roi de Naples, et sur la nourrice disant à Pierre quand il peut venir parler à Maguelonne, sections qui dans les éditions troyennes et rouennaise du XVú s. et dans celles des Trepperel et de le Roy forment un chapitre distinct, font dans les éditions Bouteiller, de La Fontaine, Didier et Chancey partie du chapitre précédent. Une division pareille en deax groupes de leçons se dessine à propos des titres des chapitres sur Pierre jetant des pierres à l'oiseau, et sur Maguelonne édifiant une église au "port sarrazin ", de même qu'à propos de la présence (groupe lyonnaise) ou absence des spécifications suivantes : Le roi voulait savoir non seulement de quel pays il venait, mais aussi de quels gens ; Pierre trouva les anneaux sur la poitrine de son amie ; Maguelonne dit à Pierre qu'elle dira à ses parents de venir à l'église le jour où son voeu finirait.

${ }^{17}$ Une même évolution semble avoir eu lieu dans Valentin et Orson, en tout cas dans le chapitre cinq, car dans l'édition du lyonnais Rigaud (1605) un des personnages s'adresse à Dieu et à la Vierge, tandis que dans l'édition de Nicolas et Pierre Bonfons (Paris, vers 1600) il n'est plus question de Marie.
} 
le style et le vocabulaire ont subi des transformations importantes. Mais malgré cela, cette édition, même si elle abrège, a conservé l'essentiel de chaque chapitre. Il n'en est pas de même de l'édition qui en cette même année 1700 sortit des presses de l'éditeur rouennais Jacques Besongne. S'inspirant d'un texte de la tradition parisienne, Besongne (ou l'éditeur du texte qui lui servait de copie ${ }^{18}$ ) a voulu réduire les frais de l'édition en utilisant seulement trois feuilles ( $48 \mathrm{pp}$.) au lieu de quatre feuilles (64 pp.) in- $8^{\circ}$ pour chaque exemplaire de son édition. Cela signifie qu'il a dû supprimer seize pages! Il aurait pu moderniser l'histoire en l'abrégeant en même temps, comme l'a probablement fait Jacques Oudot. Besongne, ou un de ses prédécesseurs, a cependant opté pour une solution plus simple et plus rigoureuse à la fois; il a supprimé quatre chapitres entiers et des parties d'autres chapitres. Cela a pour conséquence que le texte prend des toumures inattendues. Par exemple, le lecteur qui a laissé Pierre à la Cour du Soudan, le retrouve dormant dans l'île de Sangona.

En guise de conclusion, récapitulons les résultats de notre étude comparative en les mettant en rapport avec la définition courante de la Bibliothèque bleue. Nous avons d'abord attiré l'attention sur notre hypothèse, à savoir qu'au XVI ${ }^{\mathrm{e}} \mathrm{s}$. des éditions «bleues" de notre roman, ainsi que d'autres romans de chevalerie, s'imprimaient non seulement à Troyes et à Rouen, mais aussi à Lyon. A notre avis, la définition de la Bibliotheqque bleue devrait être révisée en ce sens. Ensuite nous avons constaté que la plupart des mutilations et cicatrices que présentent les éditions «bleues » du XVII s. n'ont pas été causées par les opérations des éditeurs de l'époque, mais par celles de leurs prédécesseurs. Cela ne vaut probablement pas seulement pour l'histoire de Pierre de Provence, mais aussi pour d'autres romans de chevalerie, sinon pour bon nombre de textes médiévaux figurant dans la Bibliothèque bleue. En effet les différentes formes que revêt notre texte au XVu' $s$. s'avèrent être le résultat d'un long processus auquel les éditeurs de la Bibliothèque bleue ont contribué d'une façon qui ne diffère guère de celle de leurs prédécesseurs des $X V^{e}$ et $X V{ }^{e}{ }^{e}$ siècles. C'était le même désir de produire à moindres frais des livres à large diffusion qui animait les éditeurs de romans de chevalerie et autres livres bleus, et leurs prédécesseurs. Cette conclusion, nous l'avons dit, n'est pas nouvelle, mais jusqu'ici elle n'a pas eu de conséquences pour la définition du concept de la Bibliothèque bleue. Appliquer ce nom à chaque publication qui est une réédition à bon marché d'un titre à succès ne nous avancerait évidemment pas. Cela n'a pourtant pas de sens non plus de dire que certaines éditions des Oudot appartiennent à cette « collection », et d'en exclure sans explication celles de leurs prédécesseurs et contemporains qui présentent les mêmes caractéristiques matérielles. Malheureusement, le fait même qu'il s'agit d'un processus continu gêne toute tentative de déterminer le niveau de "dégradation " que doit posséder un livre pour faire partie de la Bibliothèque bleue, et même tout essai de définition. Seuls des travaux de comparaison approfondis et portant sur plusieurs textes différents pourraient peut-être mettre en avant des critères précis. Donc plutôt que de proposer ici une nouvelle définition, plus élaborée, nous devons nous borner à problématiser la définition utilisée jusqu'ici.

\footnotetext{
${ }^{18}$ Il pourrait avoir eu sous les yeux l'édition de David Ferrand, ou peut-être celle de N. et P. Bonfons.
} 


\section{La réception productive de Pierre de Provence; antibibliothèques et poésies galantes}

Dans la littérature du Xvif $\mathrm{s}$., les allusions aux romans de chevalerie médiévaux sont nombreuses. Nous nous arrêterons ci-dessous à deux groupes d'allusions à Pierre de Provence, trahissant, pour les romans de chevalerie, deux modes de réception importants à l'époque. Le premier groupe consiste en références visant à faire rire le public, soit en ridiculisant le genre du "vieux roman", lecture des ignorants, soit en évoquant les vieux titres dans un contexte comique. Ces allusions prennent souvent la forme d'énumérations plus ou moins longues, où figurent plusieurs représentants du genre. Voici quelques titres de la bibliothèque d'un poète pauvre, qui, en dressant l'inventaire de ses biens, veut convaincre le roi qu'il n'a pas d'argent pour payer la taille : «Geoffroy, dis la grand'dent, les quatre fils d'Aymon, /Le Compost des Bergers, \& Robert le Demon;[...] Gallien retoré, Alexis Piedmontois, $\mathrm{L}$ a Flacon qui remet les veillards en jouuence, /La belle Maguelonne, \& Pierre de Prouence, $/$ [...] Ollivier le Dannois, Valentin et Orson. ${ }^{19}$ Les bateleurs célèbres comme Tabarin et Bruscambile avaient souvent recours aux romans de chevalerie pour exciter l'hilarité de leur public. Par exemple, Tabarin demande un jour à son compagnon, «le maistre», qui a étudié les chroniques, de quand date la mode de "froncer les chemises». Celui-ci répond que les annales ne contiennent pas d'informations sur ce genre de trivialités, mais qu'elles racontent les faits et gestes des «grands personnages» de l'histoire, ce qui amène Tabarin à tirer la conclusion suivante : «Je prendray donc le roman de Jean de Paris, de Regnaut de Montauban, de la belle Maguelonne, de Richard-sans-Peur, et autres infinies histoires, pour annales : car vous ne vistes jamais plus beaux faicts d'armes ny courages plus hardis. ${ }^{20}$

Le deuxième groupe est constitué par des références à l'amour de Pierre et Maguelonne qui, sous la plume des écrivains mondains du XVII $\mathrm{e}$., est dépourvu de son caractère chaste et pieux et prend les allures d'un amour galant à la mode; car si le XVII' s. n'était jamais las de chanter et d'entendre les exploits des héros nationaux, qu'ils soient personnages historiques ou personnages de roman, il pensait que le parfait chevalier était aussi galant que vaillant. Le contenu des rondeaux que s'amusait à faire pendant quelque temps le beau monde en témoigne, entre autres celui des quatre «rondeaux antiques » sur lesquels s'ouvre le Nouveau recueil de divers mondeaux (1650), et qui sont intitulés Pour Richard sans Peur, Pour Pierne de Provence, Pour Galien Restauré, Pour Ogier le Danois. Bien que la date de leur composition ne soit pas connue, on assume qu'ils datent de la fin du XVI ${ }^{e}$ s. au plus tôt. ${ }^{21}$ Voici le demier couplet du rondeau de Pierre de Provence : «Francs Cheualiers, qui parmy

\footnotetext{
19 «Requeste du poëte de campagne au Prince de Monopolie ", Euvres galantes de monsieur Cotin, Paris, E. Loyson, 1665, p. 429. Pour d'autres exemples cf. M. Simonin, «La réputation des romans de chevalerie selon quelques listes de livres ( $\mathrm{xV}^{e}-\mathrm{xVII}$ siécles) ", Mélanges de langue et littérature françaises du Moyen Age et de la Renaissance, offerts à M. Ch Foulon, Rennes, 1980, t. 1, pp. 363369.

${ }^{20}$ Euvres completes de Tabarin [...] par G. Aventin, Paris, P. Jannet, 1858, t. 1, p. 173.

${ }^{21}$ Cf. Euvres de La Bruyère. Nouvelle édition [...] par M. G. Servois, Paris, Hachette et Cie, 1865, t. 2, p. 216.
} 
vos amours/Souffrés méhaim, traverses, \& clamours,/Souuenez-vous de Pierre de Prouence;/Ainsi que luy, ioye, soulas, respitD'amour aurez, s'auez bonne constance,/Malgré fortune, \& mesmes en dépit/De tout le monde. ${ }^{22}$ En 1638, Gaston d'Orléans, qui voulait divertir une jeune femme dont il était amoureux, fit préparer un somptueux ballet qui reçut le titre de Ballet du mariage de Pierre de Provence et de la belle Maguelonne. Gaston et son amie interprétaient évidemment les rôles de Pierre et Maguelonne. L'on trouve peu de traces du roman médiéval dans l'action du ballet, qui se laisse facilement résumer: Pierre étant parti pour accomplir des faits d'armes, Maguelonne, qui s'ennuye, décide d'aller à Tours (résidence de G. d'Orléans) où se trouve en ce moment la cour de Niflezest, reine des Andouilles (!). Après un certain temps, Pierre s'y rend aussi, accompagné d'un train magnifique. Les courtisans de Niflezest, les filles d'honneur de Maguelonne et les personnages accompagnant Pierre récitent des vers où règnent l'esprit galant le ton burlesque de la cour de Monsieur. Ainsi Niflezest fait l'éloge de Bacchus, Cerès et l'Abondance, deux "muletiers yvres" se plaignent de ce que leurs animaux de bagages ne peuvent pas suivre leurs pas titubants, et Pierre annonce: «Amour d'un de ses dards a percé ma poictrine,/Pour un jeune sujet, beau sans comparaison;/Mais si dans peu de temps il ne m'en fait raison,Je pourrai bien briser son arc sur son eschine. " Après que Pierre a fait preuve de sa valeur dans plusieurs toumois, Maguelonne, pour montrer son affection pour lui, danse un ballet. Finalement, la reine Niflezest marie les deux amants et le ballet se termine par ces vers adressés par Maguelonne aux dames de la cour: "Chastes Beautez, dont les rigueurs/Laissent consumer tant de coeurs,/C'est assez par la flamme esprouver leur constance./Aymez, à mon exemple, en cet aymable jour/Où mon fidelle amant par sa perseverance/Doit enfin moisonner les fruicts de son amour. ${ }^{23}$ Peu après la représentation de ce ballet, Louison Roger de la Marbellière reçut le titre officiel de "Maitresse de Monsieur $"{ }^{24}$ C'est sans doute aussi un amour qui n'est ni chaste ni pieux auquel se réfère l'auteur anonyme d'un catalogue de livres fictifs visant à critiquer ses contemporains, catalogue qui recense Les Amours de la belle Magdelaine et de Pierre de Provence, commentées par Monseigneur de Paris, dédiées à Mad. de Lesdiguières. ${ }^{25}$ Il s'agit ici de l'affaire (présumée) de François de Harlay, archevêque de Paris et grand amateur de femmes, et Paule-Françoise-Marguerite de Gondi, veuve du duc de Lesdiguières. ${ }^{26}$

\footnotetext{
${ }^{22}$ Nouveau recueil de divers rondeaux, Paris, A. Courbé, 1650, p. 3.

${ }^{23}$ "Ballet du mariage de Pierre de Provence et de la belle Maguelonne", S.1., 1638, Ballets et mascanades de cour de Henri Il à Louis XIV (1581-1652), recueillis et publiés par P. Lacroix, Genève, Slatkine, 1968, t.5, pp. 181-197. [Réimpression de l'édition de Genève, 1868-1870.]

${ }^{24}$ Cf. G. Dethan, Gaston d'Orléans, conspirateur et prince chamant, Paris, Fayard, 1959, pp. 195-196. Le ballet a d'ailleurs remporté d'autres succès ; après avoir été exécuté à l'hôtel de Monsieur, il fut remanié et dansé dans la grande salle du palais de Tours et ensuite réimprimé par Cardin Besongne. On en parla tant à la cour que le cardinal de Richelieu dernanda à le représenter pour lui et pour le roi, ce qui fut fait. Robert Quinet réimprima alors le livret, qui à cette occasion reçưt le titre de Grand ballet de Monsieur. Cf. P. Lacroix, op. cit, p. 181.

${ }_{25}$ "Catalogue de livres nouveaux, 1693, à Paris. ", E. Griselle, "Curiosités bibliographiques ", Bulletin $d u$ Bibliophile et du Bibliothécaire, 75, 1911, p. 182.

${ }^{26}$ Sur les rumeurs qui couraient à propos de ces amants présumés cf. CEuvres de La Bruyère, op. cit. t. 2, p. 45, 300, et Mémoines de Saint Simon. Nouvelle édition [...J par A. de Boislisle, Paris, Hachette et Cie, 1879, t. 2, pp. 350-351.
} 
Ces allusions de natures diverses ${ }^{27}$ nous montrent que le roman de Pierre de Provence, ainsi que d'autres romans de chevalerie médiévaux, était toujours bien connu au XVII s., non seulement du peuple, lecteur d'éditions «bleues» selon Gariel ${ }^{28}$, mais aussi des esprits cultivés. Elles nous apprennent qu'en plein siècle classique les romans chevaleresques faisaient partie du bagage culturel des Français et qu'ils n'hésitaient pas à s'en servir. Mais tandis que la réception matérielle de Pierre de Provence, malgré toutes les déformations dont a souffert le texte original, donne une interprétation de l'histoire qui est assez fidele aux intentions de l'auteur, la réception productive fait preuve d'une attitude moins respectueuse envers notre roman. Les auteurs de l'époque n'en retiennent souvent qu'un seul aspect qui les intéresse et cet aspect est encore adapté aux goûts et aux règles littéraires en vogue. Certaines allusions aux romans de chevalerie donnent matière à soupçonner que leurs pères spirituels connaissent seulement les titres des livres auxquels ils se réfèrent, et parfois même pas ceux-ci, car quelques titres sont cités de façon incorrecte, comme par exemple Ollivier le Dannois, combinaison de Ogier le Danois et Ollivier de Castille, dans l'inventaire du poète pauvre. Mais qu'elles soient ridiculisées, adaptées, considérées comme documents historiques ou traitées comme œuvres littéraires dignes d'être lues, ces vieilles histoires continuent à attirer sur elles l'attention des hommes de l'époque dite classique.

L'étude de la réception de Pierre de Provence au Xvil' ${ }^{2}{ }^{29}$, qui nous montre en raccourci la réception de tout un genre, nous fait donc entrevoir des aspects peu connus et difficiles à saisir du Grand Siècle, et elle nous place devant la fascinante énigme de la force d'une littérature qui, tout comme Pierre retrouva celle qui l'aimait, à travers toutes sortes de tribulations, a toujours su trouver des lecteurs.

\section{Helwi Blom (Université d'Utrecht)}

\footnotetext{
${ }^{27}$ Pour d'autres allusions voir entre autres Les milles imaginations de Cypille [...], Par le sieur de $\mathbf{M}$. seigneur de P., Paris, A. Saugrain, 1609, p. 51, et Les souffleurs, comedie, Paris, Veuve Ch. Coignard, 1694, pp. 45-48.

${ }^{28} \mathrm{La}$ question du public de la Bibliothéque bleue, que nous ne pouvons pas aborder ici, reste un des problèmes les plus pénibles à résoudre. Nous pensons qu'en suggérant que les lecteurs de la 'collection'se recrutent dans les couches les plus basses de la population (urbaine ?), Gariel donne une image tendencieuse des lecteurs de 'vieux romans'.

${ }^{29}$ Nous remercions vivement l'Institut des Recherches Historiques et Culturelles de I'Université d'Utrecht, ainsi que l'Organisation Nëerlandaise pour la Recherche Scientifique, qui nous ont foumi les moyens pour un séjour de travail à Paris.
} 